\title{
Coupling between MRI-assessed regional aortic pulse wave velocity and diameters in patients with thoracic aortic aneurysm: a feasibility study
}

\author{
E. S. J. Kröner · J. J. M. Westenberg • \\ L. J. M. Kroft • N. J. Brouwer • \\ P. J. van den Boogaard • A. J. H. A. Scholte
}

Published online: 24 July 2015

(C) The Author(s) 2015. This article is published with open access at Springerlink.com

\begin{abstract}
Aims Thoracic aortic aneurysm (TAA) is potentially lifethreatening and requires close follow-up to prevent aortic dissection. Aortic stiffness and size are considered to be coupled. Regional aortic stiffness in patients with TAA is unknown. We aimed to evaluate coupling between regional pulse wave velocity (PWV), a marker of vascular stiffness, and aortic diameter in TAA patients.

Methods In 40 TAA patients ( $59 \pm 13$ years, 28 male), regional aortic diameters and regional PWV were assessed by 1.5 T MRI. The incidence of increased diameter and PWV were determined for five aortic segments (S1, ascending aorta; S2, aortic arch; S3, thoracic descending aorta; S4, suprarenal and S5, infrarenal abdominal aorta). In addition, coupling between regional PWV testing and aortic dilatation was evaluated and specificity and sensitivity were assessed. Results Aortic diameter was $44 \pm 5 \mathrm{~mm}$ for the aortic root and $39 \pm 5 \mathrm{~mm}$ for the ascending aorta. PWV was increased in $36(19 \%)$ aortic segments. Aortic diameter was increased in $28(14 \%)$ segments. Specificity of regional PWV testing for the prediction of increased regional diameter was $\geq 84 \%$ in the descending thoracic to abdominal aorta and $\geq 68 \%$ in the ascending aorta and aortic arch.
\end{abstract}

E. S. J. Kröner $(\bowtie) \cdot$ N. J. Brouwer · A. J. H. A. Scholte Department of Cardiology, Leiden University Medical Center, Leiden, The Netherlands

e-mail: e.s.j.kroner@lumc.nl

\section{E. S. J. Kröner}

The Interuniversity Cardiology Institute of the Netherlands, Utrecht, The Netherlands

J. J. M. Westenberg · L. J. M. Kroft · P. J. van den Boogaard Department of Radiology, Leiden University Medical Center, Leiden, The Netherlands
Conclusion Normal regional PWV is related to absence of increased diameter, with high specificity in the descending thoracic to abdominal aorta and moderate results in the ascending aorta and aortic arch.

Keywords Thoracic aortic aneurysm - Aortic dilatation · Aortic stiffness $\cdot$ MRI

\section{Introduction}

Thoracic aortic aneurysm (TAA) is a life-threatening disease which requires close follow-up to intervene before aortic dissection and/or rupture occurs. The incidence of aortic disease increases and has been estimated at 10.4 cases per 100,000 person/years [1, 2]. A TAA grows asymptomatically and slowly, at approximately $0.1-0.42 \mathrm{~cm}$ per year [3-5]. Besides the increase in aortic diameter, mechanical properties of the aneurismal aorta deteriorate [6]. Assessment of the mechanical properties of the aorta (i.e. aortic stiffness) may be useful to supplement symptoms and size as criteria for prophylactic aortic intervention [7].

Aortic pulse wave velocity (PWV), a well-validated surrogate marker of arterial stiffness, is defined as the propagation speed of the systolic velocity wave front through the aorta [8]. PWV assessment by magnetic resonance imaging (MRI), using velocity encoding allows for full threedimensional visualisation of the aorta, thereby permitting reproducible positioning of imaging planes along the aorta [9]. Moreover, MRI allows for the assessment of global, regional and local PWV at different locations in the arterial tree [8]. PWV assessment from velocity-encoded MRI, when compared directly with PWV assessment from invasive pressure measurements (the gold standard), had excellent correlation and reproducibility $[8,10]$. 
In patients with Marfan syndrome, increased global and regional aortic wall stiffness has been reported [11]. Furthermore, PWV in the aortic arch showed statistically significantly more increase with age, suggesting more pronounced aortic involvement in the proximal aorta [11]. This is also a possible risk factor for aortic dissection and therefore regional PWV assessment can be of clinical value in patients with TAA. It is expected that in TAA patients without Marfan syndrome, local aortic wall stiffness measured by $\mathrm{PWV}$ is increased. Interestingly, regional aortic wall stiffness and size are considered to be coupled [6, 12]. However, the local aortic wall stiffness, measured by PWV in TAA patients, has not been reported before. Therefore, the purpose of the present study was (1) to investigate the local aortic wall stiffness measured by PWV in TAA patients and (2) to evaluate coupling between regional PWV and aortic diameter and (3) to assess sensitivity and specificity for the coupling between regional PWV testing and aortic dilatation in TAA patients.

\section{Methods}

Patient population and study protocol

The study population consisted of 40 adult TAA patients. Selection criteria were: (1) age between 18-80 years, (2) asymptomatic for aortic dilatation and/or dissection and (3) regularly clinically evaluated at the outpatient clinic, including MRI examination of the entire aorta. Exclusion criteria were: (1) Marfan syndrome and related disorders (left out by clinical evaluation and/or DNA analysis) and (2) history of aortic surgery, significant aortic valve or mitral valve regurgitation.

All patients underwent a comprehensive MRI examination to assess both regional aortic lumen diameter and regional aortic stiffness (PWV), by using velocity-encoded MRI. Patients temporarily refrained from beta-adrenergic blocking medication and were at least $24 \mathrm{~h}$ without this medication prior to MRI.

First, the regional aortic maximal diameter and regional PWV were compared against age-related normal values and the incidence of increased aortic diameter and/or increased segmental PWV was determined. Second, sensitivity and specificity for the coupling between regional PWV testing and aortic dilatation in TAA patients were assessed. The study was conducted with the approval of the Leiden University Medical Center Institutional Review Board with a specific waiver of the need for individual patient consent.

MRI acquisition

MRI acquisition was performed with a $1.5 \mathrm{~T}$ scanner (Philips Intera, release 12; Philips Medical Systems, Best, the Neth- erlands; pulsar gradient system with amplitude $33 \mathrm{mT} / \mathrm{m}$, $100 \mathrm{mT} / \mathrm{m} / \mathrm{ms}$ slew rate, and $0.33 \mathrm{~ms}$ rise time).

\section{Acquisition of aortic dimensions}

Aortic dimensions were assessed from the acquisition of conventional (1) cine bright-blood gradient-echo imaging in coronal (Fig. 1a) and sagittal (Fig. 1b) direction and (2) twodimensional black-blood spin-echo imaging (Fig. 1c). To visualise the entire aorta, a three-dimensional, T1-weighted fast gradient-echo sequence was used to obtain a contrastenhanced magnetic resonance angiography (MRA) of the full aorta, as previously described (Fig. 1d) [12].

\section{Acquisition of aortic PWV}

Regional PWV was assessed by two consecutive three-slice two-directional in-plane velocity-encoded MRI acquisitions as described before [12]. Velocity encoding was performed in phase-encoding (i.e., anterior-posterior) direction and in frequency-encoding (i.e., feet-head) direction consecutively. The velocity sensitivity was set to $150 \mathrm{~cm} / \mathrm{s}$ and we used scan parameters as described earlier [12]. In short, $60 \%$ rectangular field-of-view (FOV) $450 \times 270 \mathrm{~mm}^{2}$, $10 \mathrm{~mm}$ slice thickness, echo time (TE) $2.4 \mathrm{~ms}$, repetition time (TR) $4.3 \mathrm{~ms}$, flip angle $\alpha 10^{\circ}$, acquisition voxel size $3.5 \times 2.1 \times 10.0 \mathrm{~mm}^{3}$, sampling bandwidth $495 \mathrm{~Hz}$ and number of signal averages (NSA) 2. Retrospective gating was performed with the maximal number of phases reconstructed. The true temporal resolution amounted to $8.6 \mathrm{~ms}$ (equals $2 \times \mathrm{TR}$ ). Acquisition was performed with free breathing. Mean scan time of a single acquisition was $7 \mathrm{~min}$ and $8 \mathrm{~s}$ at a typical heart rate of 65 beats $/ \mathrm{min}$.

Image analysis

A schematic representation of image acquisition and analysis for aortic diameter and aortic PWV in a TAA patient is provided in Fig. 1 and 2, respectively. The aorta was divided into five segments; the ascending aorta (S1), which included the aortic root, the tubular portion of the ascending aorta and extending to the brachiocephalic artery origin; the aortic arch (S2), which begins at the origin of the brachiocephalic artery, extending to the left subclavian artery; the thoracic descending aorta (S3) which begins at the left subclavian artery extending to the level of the diaphragm; the suprarenal abdominal aorta (S4) from the level of the diaphragm to the origin of the renal arteries; the infrarenal abdominal aorta (S5) (Fig. 1).

\section{Analysis of aortic dimensions}

Maximal diameters were evaluated on the conventional cine gradient-echo and spin-echo images at the level of the aor- 


\title{
Advertisement placed here.
}

\author{
CCS Bohn

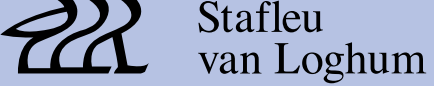 \\ Springer Media
}

Houten 2015 


\section{Advertisement placed here.}

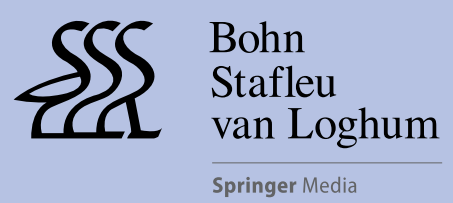

Houten 2015 

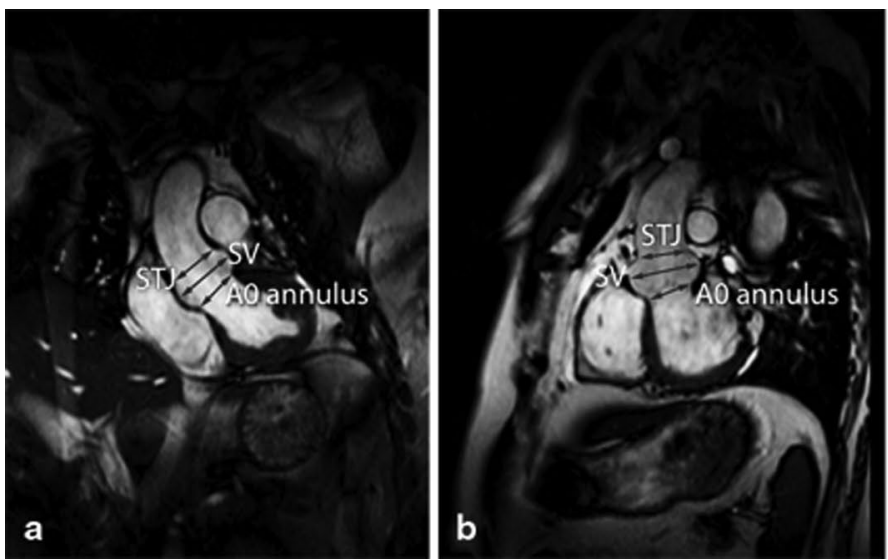

Fig. 1 Representation of image acquisition and analysis of aortic dimensions in a TAA patient. $\mathbf{a}$ and $\mathbf{b}$ are acquired by conventional cine bright blood gradient-echo imaging. $\mathbf{a}$ is taken in the oblique sagittal plane and $\mathbf{b}$ in the oblique coronal plane. $\mathbf{a}$ and $\mathbf{b}$ show the levels of the aortic annulus (Ao annulus), sinuses of Valsalva $(S V)$ and sino-tubular junction $(S T J)$ were maximal luminal diameters were evaluated. $\mathbf{c}$ is acquired by $2 \mathrm{D}$ black blood spin-echo imaging and shows the levels of the ascending aorta $(A A)$, aortic arch $(A r c h)$ and thoracic descending aorta $(D A)$ were maximal aortic diameters were measured. $\mathbf{d}$ is a maxi-

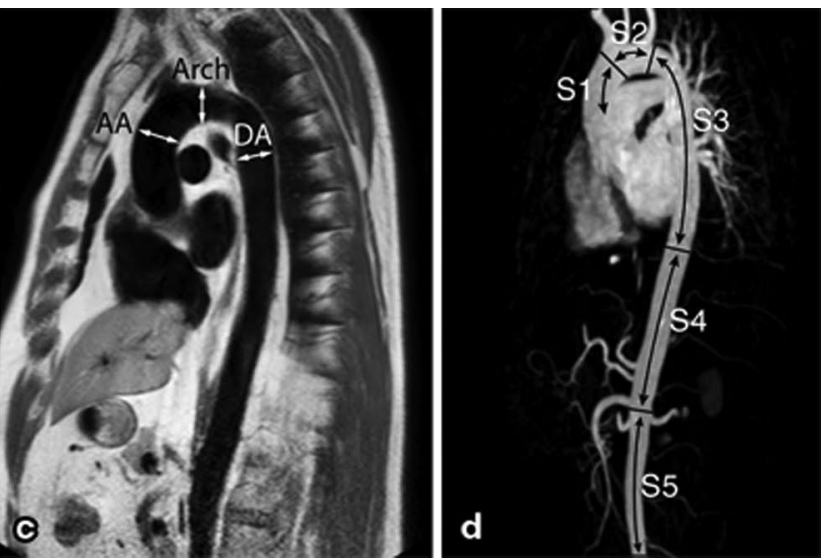

mum intensity projection of a contrast-enhanced magnetic resonance angiogram, acquired by first-pass imaging with a three-dimensional, T1-weighted fast gradient-echo sequence, representing the entire aorta. For both analysis of aortic dimensions and for analysis of aortic PWV, five aortic segments were evaluated: the ascending aorta $(S 1)$, the aortic arch $(S 2)$, the thoracic descending aorta $(S 3)$, the suprarenal abdominal aorta (S4), the infrarenal abdominal aorta (S5). Ao aorta, $S V$ sinuses of Valsalva, $S T J$ sino-tubular junction, $A A$ ascending aorta, $D A$ descending aorta
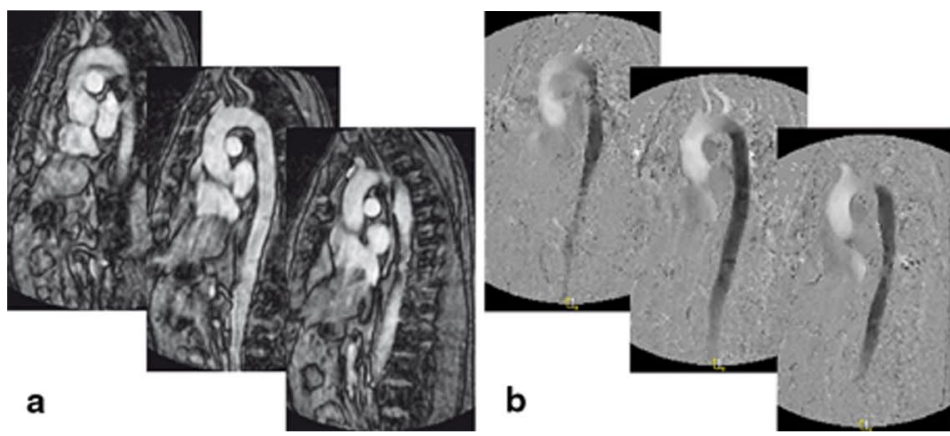
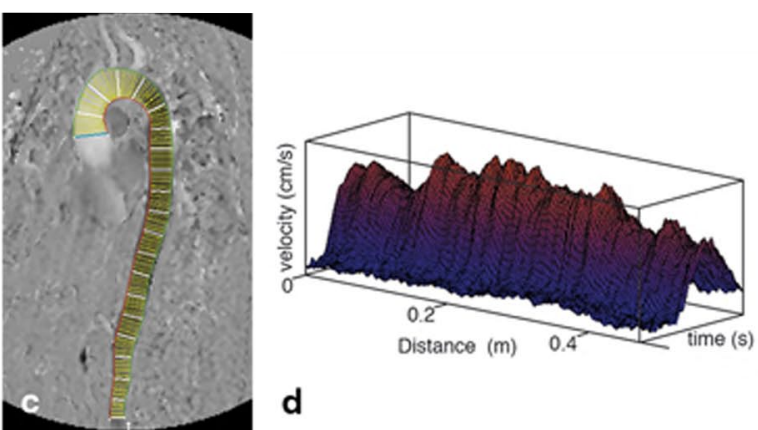

d
Fig. 2 Representation of image acquisition and analysis of regional PWV in a TAA patient. Regional PWV was assessed by means of combining two consecutive acquisitions of a stack of three consecutive slices (a), both with one-directional velocity-encoding in anteriorposterior and feet-head direction (b), respectively. The stack of three slices covered the entire aorta. Using MASS MRI software, 200 equidistantly-spaced sampling chords were defined (c). At each sampling

tic annulus, aortic root, sino-tubular junction, the thoracic ascending aorta, the aortic arch, and the thoracic descending aorta. An example is provided in Fig. 1a-d. The aortic dimensions in TAA patients were considered increased if the values exceeded the criteria as described in the guidelines for the diagnosis and management of patients with thoracic aortic segments [13]. In detail, for the aortic root, aortic enlargement was quantified based on age- and body size-adjusted Roman criteria [13, 14]. For segment 1, the age- and body size-adjusted Hannuksela criteria were used $[13,15]$, for segment 2 , a cut off of $30 \mathrm{~mm}$ was used, based on the guidelines [13], for segment 3 , again, the age- and body size-adjusted Hannuksela criteria were used [13, 15]. chord, the maximal velocity along the aortic centreline was determined for each cardiac phase, resulting in 200 velocity-time waveforms (d). From the corresponding measurement position (distance $(\mathrm{x})$ ) along the aortic centreline and time of arrival of the pulse wave (arrival time (t)) at each position, the local aortic pulse wave velocity was determined. $P W V$ pulse wave velocity, TAA thoracic aortic aneurysm, $V E$ velocityencoded, $M R I$ magnetic resonance imaging

And for segment 4 and 5, aortic dilatation was diagnosed when the aorta exceeds a generally agreed to standard diameter or when a given aortic segment was larger than contiguous aortic segments of apparently normal size, according to the guidelines [13].

Mean diameter per aortic segment was obtained from the contrast-enhanced MRA data, using in-house developed and validated software for automated vessel analysis (LAVA software) [15]. 
Analysis of aortic PWV

Regional PWV was obtained from the velocity-encoded MRI data, using in-house developed MASS software [10]. An example is provided in Fig. 2a-d. After manual segmentation of the aorta, the aortic centreline was automatically determined and 200 equidistantly-spaced sampling chords were defined. For each chord and each phase the maximal velocity wave form was determined, resulting in 200 maximal velocity waveforms $[10,12,16]$. The arrival time (time (t)) of each of the 200 waveforms at their corresponding positions (distance (x)) along the aortic centreline was automatically determined and PWV, defined as $\Delta \mathrm{x} / \Delta \mathrm{t}$, was calculated $[10,12,16]$. This regional PWV was obtained for each of the five aortic segments.

Consecutively, regional PWV values from the TAA patients were compared with age-related normal values (using linear regression: $\mathrm{PWV}=\mathrm{A} \times \mathrm{Age}+\mathrm{B}$ ) from a healthy volunteer cohort [12]. The PWV was considered increased if this value exceeded the predicted normal PWV with two standard errors (SE) for each of the regression coefficients $\left(\mathrm{PWV}>\left(\left(\mathrm{A}+2 \times \mathrm{SE}_{\mathrm{A}}\right) \times \mathrm{AGE}+\left(\mathrm{B}+2 \times \mathrm{SE}_{\mathrm{B}}\right)\right)\right.$.

\section{Statistical analysis}

Continuous variables are expressed as mean \pm standard deviation (SD). Sensitivity and specificity were calculated for the coupling between regional aortic PWV assessment and regional aortic dilatation in TAA patients. Statistical analysis was performed using SPSS v 20.0 (SPSS Inc., Chicago, IL).

\section{Results}

Forty patients (59 \pm 13 years) were evaluated. Patient characteristics at the time of inclusion are summarised in Table 1 . Twenty-eight patients $(70 \%)$ were male. Patients were often hypertensive or used antihypertensive medication $(n=27,68 \%)$.

Aortic diameter

Maximal aortic diameter was $27 \pm 3 \mathrm{~mm}$ for the aortic annulus, $44 \pm 5 \mathrm{~mm}$ for the aortic root and $36 \pm 5 \mathrm{~mm}$ for the sinotubular junction. For the aortic segments, mean and maximal aortic diameter dimensions are provided in Table 2.

PWV values

Mean regional PWV values are presented in Table 2. A total of 194 aortic segments were evaluated. Of note, 6 aortic segments ( $1 ; n=1, \mathrm{~S} 2 ; n=3 ; \mathrm{S} 5 ; n=2)$ were considered
Table 1 Demographic and clinical characteristics of TAA patients $(n=40)$

\begin{tabular}{|c|c|}
\hline \multicolumn{2}{|l|}{ Demographics } \\
\hline Male/female & $28(70 \%) / 12(30 \%)$ \\
\hline Age at MRI (years) & $59 \pm 13$ \\
\hline \multicolumn{2}{|l|}{ Clinical characteristics } \\
\hline Height $(\mathrm{cm})$ & $180 \pm 10$ \\
\hline Body mass index $\left(\mathrm{kg} / \mathrm{m}^{2}\right)$ & $27 \pm 4$ \\
\hline Body surface area $\left(\mathrm{m}^{2}\right)$ & $2.1 \pm 0.2$ \\
\hline \multicolumn{2}{|c|}{ Brachial blood pressure ( $\mathrm{mmHg}$ ) } \\
\hline Systolic & $134 \pm 20$ \\
\hline Diastolic & $79 \pm 11$ \\
\hline Heart rate (beats/minute) & $66 \pm 10$ \\
\hline \multicolumn{2}{|c|}{ MRI assessed LV dimensions and function } \\
\hline $\mathrm{EDV}(\mathrm{ml})$ & $164 \pm 38$ \\
\hline $\operatorname{EDV} / \mathrm{BSA}\left(\mathrm{ml} / \mathrm{m}^{2}\right)$ & $80 \pm 19$ \\
\hline $\mathrm{ESV}(\mathrm{ml})$ & $69 \pm 23$ \\
\hline $\operatorname{ESV} / \mathrm{BSA}\left(\mathrm{ml} / \mathrm{m}^{2}\right)$ & $34 \pm 12$ \\
\hline Ejection fraction $(\%)$ & $62 \pm 5$ \\
\hline \multicolumn{2}{|l|}{ Cardiovascular risk factors } \\
\hline Hypertension $^{\mathrm{a}}$ & $27(68 \%)$ \\
\hline Current smoker & $7(18 \%)$ \\
\hline Hypercholesterolaemia $^{\mathrm{b}}$ & $16(40 \%)$ \\
\hline Diabetes mellitus & $1(3 \%)$ \\
\hline Obesity $^{\mathrm{c}}$ & $7(18 \%)$ \\
\hline Previous myocardial infarction & $4(10 \%)$ \\
\hline \multicolumn{2}{|l|}{ Cardiovascular medication } \\
\hline$\beta$-blocker & $17(58 \%)$ \\
\hline ACE inhibitor & $21(53 \%)$ \\
\hline Anticoagulants & $16(40 \%)$ \\
\hline Statins & $13(33 \%)$ \\
\hline
\end{tabular}

Data are presented as number (percentage) or as mean \pm standard deviation.

$L V$ left ventricular, $E D V$ end-diastolic volume, $E S V$ end-systolic volume, $B S A$ body surface area.

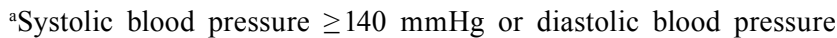
$\geq 90 \mathrm{mmHg}$ or use of antihypertensive medication.

${ }^{\text {bSerum }}$ total cholesterol $\geq 230 \mathrm{mg} / \mathrm{dl}$ or serum triglycerides $\geq 200 \mathrm{mg}$ / $\mathrm{dl}$ or treatment with lipid-lowering drugs.

${ }^{\mathrm{c}}$ Body mass index $\geq 30 \mathrm{~kg} / \mathrm{m}^{2}$.

failed ( $3 \%)$, potentially due to excessive patient movement, and therefore not included in the analysis.

Coupling between PWV and aortic diameters

The incidence of increased aortic dimensions and the incidence of increased PWV are presented in Table 3. Regional PWV was increased in 36 (19\%) aortic segments (S1: 8 cases, S2: 11 cases, S3: 7 cases, S4: 6 cases, S5: 4 cases). In total, 18 (45\%) TAA patients presented with increased regional $\mathrm{PWV}$ in $\geq 1$ aortic segment. The aortic root was increased in $38(95 \%)$ out of the 40 TAA patients. For the five aortic segments distal to the aortic root, in $21(53 \%)$ out of the 40 patients regional aortic diameter was increased in at least one aortic segment. Regional aortic diameter was 
Table 2 Aortic dimensions and PWV per aortic segment $(n=40)$

\begin{tabular}{lllll}
\hline Aortic segment & $\begin{array}{l}\text { Diameters }(\text { maximal luminal } \\
\text { diameter, } \mathrm{mm})\end{array}$ & $\begin{array}{l}\text { Diameters (mean luminal } \\
\text { diameter, mm) }\end{array}$ & PWV (m/s) & $\begin{array}{l}\text { PWV, Number of } \\
\text { patients analysed }\end{array}$ \\
\hline S1: Ascending aorta & $39.4 \pm 5.3$ & $34.8 \pm 4.2$ & $7.7 \pm 4.8$ & 39 \\
S2: Aortic arch & $28.7 \pm 3.2$ & $29.4 \pm 2.7$ & $7.7 \pm 5.6$ & 37 \\
S3: Thoracic descending aorta & $26.6 \pm 5.7$ & $24.3 \pm 2.7$ & $8.5 \pm 3.9$ & 40 \\
S4: Suprarenal abdominal aorta & $25 \pm 3$ & $22.4 \pm 2.6$ & $8.3 \pm 4.0$ & 40 \\
S5: Infrarenal abdominal aorta & $18.5 \pm 3.2$ & $17.2 \pm 3.7$ & $8.3 \pm 4.0$ & 38 \\
\hline
\end{tabular}

Data are presented as mean \pm standard deviation.

$P W V$ pulse wave velocity, $S 1$ ascending aorta, $S 2$ aortic arch, $S 3$ thoracic descending aorta, $S 4$ suprarenal abdominal aorta, $S 5$ infrarenal abdominal aorta.

Table 3 Diagnostic performance (i.e. sensitivity and specificity) of regional PWV testing in predicting regional aortic dilatation

\begin{tabular}{|c|c|c|c|c|c|c|c|}
\hline \multirow[t]{2}{*}{$N$} & \multirow[t]{2}{*}{ Aortic Segment } & \multicolumn{2}{|c|}{ Incidence of increased } & \multirow{2}{*}{$\begin{array}{l}\text { Sensitivity } \\
\text { (CI) }\end{array}$} & \multirow{2}{*}{$\begin{array}{l}\text { Specificity } \\
\text { (CI) }\end{array}$} & \multirow{2}{*}{$\begin{array}{l}\text { Positive predic- } \\
\text { tive value }(\mathrm{CI})\end{array}$} & \multirow{2}{*}{$\begin{array}{l}\text { Negative predic- } \\
\text { tive value }(\mathrm{CI})\end{array}$} \\
\hline & & PWV & Diameter & & & & \\
\hline 39 & S1: Ascending aorta & $8(21 \%)$ & $15(38 \%)$ & $\begin{array}{l}13 \% \\
(2-42 \%)\end{array}$ & $\begin{array}{l}75 \% \\
(53-89 \%)\end{array}$ & $\begin{array}{l}25 \% \\
(4-64 \%)\end{array}$ & $\begin{array}{l}58 \% \\
(39-75)\end{array}$ \\
\hline 37 & S2: Aortic arch & $11(28 \%)$ & $6(16 \%)$ & $\begin{array}{l}17 \% \\
(1-64 \%)\end{array}$ & $\begin{array}{l}68 \% \\
(49-83 \%)\end{array}$ & $\begin{array}{l}9 \% \\
(0-43)\end{array}$ & $\begin{array}{l}81 \% \\
(60-93)\end{array}$ \\
\hline 40 & S3: Thoracic descending aorta & $7(18 \%)$ & $4(10 \%)$ & $\begin{array}{l}75 \% \\
(22-99 \%)\end{array}$ & $\begin{array}{l}89 \% \\
(73-96 \%)\end{array}$ & $\begin{array}{l}43 \% \\
(12-80 \%)\end{array}$ & $\begin{array}{l}97 \% \\
(82-100 \%)\end{array}$ \\
\hline 40 & S4: Suprarenal abdominal aorta & $6(15 \%)$ & $2(5 \%)$ & $\begin{array}{l}0 \% \\
(0-80 \%)\end{array}$ & $\begin{array}{l}84 \% \\
(68-93 \%)\end{array}$ & $\begin{array}{l}0 \% \\
(0-48 \%)\end{array}$ & $\begin{array}{l}94 \% \\
(79-99 \%)\end{array}$ \\
\hline 38 & S5: Infrarenal abdominal aorta & $4(10 \%)$ & $1(3 \%)$ & $\begin{array}{l}100 \% \\
(5-100 \%)\end{array}$ & $\begin{array}{l}92 \% \\
(77-98 \%)\end{array}$ & $\begin{array}{l}25 \% \\
(1-78 \%)\end{array}$ & $\begin{array}{l}100 \% \\
(87-100 \%)\end{array}$ \\
\hline
\end{tabular}

Data are presented as number (percentage).

$N$ number of patients analysed, $C I, 95 \%$ confidence interval, $P W V$ pulse wave velocity, $S 1$ ascending aorta, $S 2$ aortic arch, $S 3$ thoracic descending aorta, $S 4$ suprarenal abdominal aorta, $S 5$ infrarenal abdominal aorta.

increased in a total of $28(14 \%)$ segments (S1: 15 cases, S2: 6 cases, S3: 4 cases, S4: 2 cases, S5: 1 cases) (Table 3). Table 3 shows that the incidence of increased regional diameter was lower in the more distal aortic segments versus the more proximal aortic segments.

Table 3 shows incidence of increased PWV and diameter and the sensitivity and specificity for the coupling between PWV and diameter. Specificity was $\geq 84 \%$ in the descending thoracic to abdominal aorta and $\geq 66 \%$ in the ascending aorta and aortic arch.

Normal regional PWV demonstrated absence of increased diameter, with high specificity in the descending thoracic to abdominal aorta and moderate results in the ascending aorta and aortic arch. Of note, in general the regional PWV assessment lacked sensitivity with low to marginal results for all aortic segments.

\section{Discussion}

The main finding of the study is that normal regional PWV is related to absence of increased diameter in patients with TAA, with high specificity in the descending thoracic to abdominal aorta and moderate results in the ascending aorta and aortic arch.
Formation of TAA is influenced by the interaction of genetic and environmental factors resulting in aortic media degeneration and breakdown of elastic fibres leading to aortic dilatation and affecting aortic elasticity [13, 17-19]. The investigation of the mechanical properties of the aorta (i.e. aortic stiffness) may be useful to supplement symptoms and size as criteria for prophylactic aortic intervention [7].

Previously, aortic stiffness, measured by PWV, was an independent predictor of progressive aortic dilatation in patients with Marfan syndrome. PWV assessment from through-plane velocity-encoded MRI is widely used and allows for PWV calculation between two locations at a certain distance along the aorta [20,21]. However, changes of mechanical properties of the aorta are typically regional. Accordingly, in the present study regional aortic stiffness from in-plane velocity-encoded MRI was densely sampled at 200 positions along the aortic centreline. When compared with age-related normal values, the regional PWV was increased in $36(19 \%)$ of all studied aortic segments of these selected TAA patients. Furthermore, increased regional aortic diameter was reported in $28(14 \%)$ of the aortic segments.

Regional aortic wall stiffness and size are considered to be coupled $[6,12]$. We previously showed that regional PWV had moderate to high specificity for predicting absence of 
regional aortic luminal growth in Marfan patients [12]. To our knowledge, the incidence of increased regional aortic stiffness, as described by PWV and the coupling between aortic stiffness and regional aortic dilatation in TAA patients without Marfan syndrome, has not been reported before. In the present study, we observed that in TAA patients, regional PWV testing is associated with high specificity $(\geq 84 \%)$ for regional aortic dilatation in the descending thoracic to abdominal aorta and provided moderate results (specificity $\geq 66 \%$ ) in the ascending aorta and aortic arch. These findings imply that regional PWV assessment in TAA patients can be used to demonstrate absence of present disease (i.e. increased aortic diameter). In contrast, regional PWV testing showed low sensitivity, in particular in the ascending aorta and aortic arch, although aortic dilatation is frequently present in these segments. These findings implicate that regional PWV testing is marginal at detecting present disease. We also observed increased aortic stiffness in the more distal aortic segments, suggesting that aortic wall abnormalities extend into the entire aorta.

At present, the implication of PWV assessment for patient management remains unclear [8]. The current study suggests that aortic stiffness is coupled to aortic diameter. Confirming normal PWV values at follow-up may rule out aortic media disease including aortic stiffening and aortic dilatation. On the other hand, increased PWV in an otherwise non-dilated aortic segment may suggest impaired aortic condition and present aortic media disease, accordingly careful monitoring and treatment is then required. Ultimately, assessment of regional aortic stiffness may even demonstrate regions of the aneurysm at high risk of disruption [7]. These issues, however, are still unclear. Accordingly, our centre will prospectively follow and monitor both the structural and mechanical parameters of this cohort to provide more evidence and direction for the clinical use of regional PWV assessment in TAA patients.

\section{Limitations}

The following limitations need to be acknowledged. Our findings are based on a relatively small and healthy TAA study population (which had not yet undergone elective aortic surgery). Since the incidence of aortic dilatation of especially the abdominal aorta was low in the present study population, sensitivity and specificity values cannot be translated to all TAA patients. Our findings need to be validated in a larger study population and also in patients with more extensive disease progression. Furthermore, the age range of TAA patients extended beyond the age range of the healthy volunteer data used for obtaining age-related normal values [12]. We performed an extrapolation by linear regression to calculate age-related normal values of
PWV for cases with higher age, using the same relation as used within the age range of healthy volunteers. PWV was considered increased, when exceeding the predicted normal PWV (predicted according to the age of the patient) with two standard errors for each of the regression coefficients $\left(\mathrm{PWV}>\left(\left(\mathrm{A}+2 \times \mathrm{SE}_{\mathrm{A}}\right) \times \mathrm{AGE}+\left(\mathrm{B}+2 \times \mathrm{SE}_{\mathrm{B}}\right)\right)\right)$.

Next, accuracy of regional PWV assessment may depend on the segment length. We divided the aorta into five standard anatomical segments, with a relatively short segment 2 (i.e. aortic arch). A longer trajectory with more sampling points may potentially result in more accurate PWV assessment. Additional sources of error for the proximal aorta are the aorta curvature, the presence of branches leading to early wave reflections thereby potentially corrupting the automated definition of the foot of the pulse wave and the motion during the cardiac cycle. Additionally, in the proximal part of the aorta, breathing motion may all have effect of the accuracy of PWV assessment. These limitations may partly explain the moderate results in the ascending aorta and aortic arch, as compared with the (thoracic) descending aorta. However, the currently used technique for dense PWV sampling with in-plane velocity-encoded MRI at 200 sampling positions, allows for global, regional and local PWV assessment with high temporal resolution (i.e. $8.6 \mathrm{~ms}$ ), which is much higher than the temporal resolution of the through-plane velocity-encoded MRI technique currently used for PWV assessment in the literature [10]. Therefore, potentially our technique allows for detection of more subtle changes in local aortic stiffness.

\section{Conclusion}

In TAA patients, normal regional PWV demonstrated absence of increased diameter, with high specificity in the descending thoracic to abdominal aorta and moderate results in the ascending aorta and aortic arch.

Acknowledgments We would like to acknowledge Mr. Gerrit Kracht for the graphic design.

\section{Funding None.}

Conflict of interests None declared.

Open Access This article is distributed under the terms of the Creative Commons Attribution License which permits any use, distribution, and reproduction in any medium, provided the original author(s) and the source are credited.

\section{References}

1. Clouse WD, Hallett JW Jr, Schaff HV, et al. Acute aortic dissection: population-based incidence compared with degenerative aortic aneurysm rupture. Mayo Clin Proc. 2004;79:176-80. 
2. Clouse WD, Hallett JW Jr, Schaff HV, Gayari MM, Ilstrup DM, Melton LJ III. Improved prognosis of thoracic aortic aneurysms: a population-based study. JAMA. 1998;280:1926-9.

3. Cambria RA, Gloviczki P, Stanson AW, et al. Outcome and expansion rate of 57 thoracoabdominal aortic aneurysms managed nonoperatively. Am J Surg. 1995;170:213-7.

4. Coady MA, Rizzo JA, Hammond GL, et al. What is the appropriate size criterion for resection of thoracic aortic aneurysms? J Thorac Cardiovasc Surg. 1997;113:476-91.

5. Davies RR, Goldstein LJ, Coady MA, et al. Yearly rupture or dissection rates for thoracic aortic aneurysms: simple prediction based on size. Ann Thorac Surg. 2002;73:17-27.

6. Koullias G, Modak R, Tranquilli M, Korkolis DP, Barash P, Elefteriades JA. Mechanical deterioration underlies malignant behavior of aneurysmal human ascending aorta. J Thorac Cardiovasc Surg. 2005;130:677-83.

7. Elefteriades JA, Farkas EA. Thoracic aortic aneurysm clinically pertinent controversies and uncertainties. J Am Coll Cardiol. 2010;55:841-57.

8. Cavalcante JL, Lima JA, Redheuil A, Al-Mallah MH. Aortic stiffness current understanding and future directions. J Am Coll Cardiol. 2011;57:1511-22.

9. van der Wall EE. Crown years for non-invasive cardiovascular imaging (Part III): 30 years cardiovascular magnetic resonance. Neth Heart J. 2013;21:263-5.

10. Westenberg JJ, de Roos A, Grotenhuis HB, et al. Improved aortic pulse wave velocity assessment from multislice two-directional in-plane velocity-encoded magnetic resonance imaging. J Magn Reson Imaging. 2010;32:1086-94.

11. Westenberg JJ, Scholte AJ, Vaskova Z, et al. Age-related and regional changes of aortic stiffness in the marfan syndrome: assessment with velocity-encoded MRI. J Magn Reson Imaging. 2011;34:526-31.

12. Kroner ES, Scholte AJ, de Koning PJ, et al. MRI-assessed regional pulse wave velocity for predicting absence of regional aorta luminal growth in marfan syndrome. Int J Cardiol. 2012;167:2977-82.
13. Hiratzka LF, Bakris GL, Beckman JA, et al. 2010 ACCF/AHA/ AATS/ACR/ASA/SCA/SCAI/SIR/STS/SVM Guidelines for the diagnosis and management of patients with thoracic aortic disease: Executive summary: A report of the American College of Cardiology Foundation/American Heart Association Task Force on Practice Guidelines, American Association for Thoracic Surgery, American College of Radiology, American Stroke Association, Society of Cardiovascular Anesthesiologists, Society for Cardiovascular Angiography and Interventions, Society of Interventional Radiology, Society of Thoracic Surgeons, and Society for Vascular Medicine. Anesth Analg. 2010;111:279-315.

14. Roman MJ, Devereux RB, Kramer-Fox R, O'Loughlin J. Twodimensional echocardiographic aortic root dimensions in normal children and adults. Am J Cardiol. 1989;64:507-12.

15. Hannuksela M, Lundqvist S, Carlberg B. Thoracic aorta - dilated or not? Scand Cardiovasc J. 2006;40:175-8.

16. Kroner ES, van der Geest RJ, Scholte AJ, et al. Evaluation of sampling density on the accuracy of aortic pulse wave velocity from velocity-encoded MRI in patients with Marfan syndrome. J Magn Reson Imaging. 2012;36:1470-6.

17. Yetman AT, Graham T. The dilated aorta in patients with congenital cardiac defects. J Am Coll Cardiol. 2009;53:461-7.

18. Grewal N, DeRuiter MC, Jongbloed MR, et al. Normal and abnormal development of the aortic wall and valve: correlation with clinical entities. Neth Heart J. 2014;22:363-9.

19. Franken R, Radonic T, den Hartog AW, et al. The revised role of TGF-beta in aortic aneurysms in Marfan syndrome. Neth Heart J. 2015;23:116-21.

20. Grotenhuis HB, Westenberg JJ, Steendijk P, et al. Validation and reproducibility of aortic pulse wave velocity as assessed with velocity-encoded MRI. J Magn Reson Imaging. 2009;30:521-6.

21. Nollen GJ, Groenink M, Tijssen JG, van der Wall EE, Mulder BJ. Aortic stiffness and diameter predict progressive aortic dilatation in patients with Marfan syndrome. Eur Heart J. 2004;25:1146-52. 